\title{
Balloon-grab technique to bridge steep renal artery during endovascular thoracoabdominal aortic aneurysm repair: technical note
}

\author{
Michal Macech', Tomasz Jakimowicz', Piotr Hammer², \\ Tadeusz Grochowiecki', Slawomir Nazarewski' \\ 'Department of General, Vascular and Transplant Surgery, Medical University of Warsaw, Poland \\ ${ }^{2}$ Cook Medical Consultant, Warsaw, Poland
}

\begin{abstract}
The aim of the study is to describe an endovascular manoeuvre that can help in the cannulation and stenting of difficult renal arteries in endovascular thoracoabdominal aortic aneurysm exclusion (EVAR) with a branched stent graft.

Routinely, dedicated branch and target vessels are cannulated in antegrade fashion through a transaxillary approach. If renal arteries are steep, tortuous, and unfavourable, cannulation failure can preclude a successful endovascular procedure. In that situation, the guidewire slips off the artery. However, another guidewire and balloon can be introduced to the target vessel through femoral access. Expansion of an additional percutaneous transluminal angioplasty (PTA) balloon in the target vessel grabs the guidewire or catheter cannulated in typical fashion and prevents it from slipping off. At this point, a stiffer wire can be introduced, and the covered stent easily bridges the target vessel. The rest of the procedure is continued typically.

Expansion of an additionally introduced balloon allows the surgeon to grab the guidewire in the renal artery, thus excluding an aneurysm during EVAR. Our early experience shows that this method is effective and durable.
\end{abstract}

Key words: thoracoabdominal aneurysm, endovascular aneurysm repair, renal artery, urgent procedure, off-the-shelf device

Acta Angiol 2017; 23, 4: 159-162

\section{Introduction}

Multi-branched stent grafts (mbSG) have established a position in endovascular repair (EVAR) of thoracoabdominal aneurysm (TAAA) [I]. When first introduced, all multi-branch prostheses were custom-made devices tailored based on the patient's anatomy and required 5 to 6 weeks for manufacturing. This time frame was not acceptable in urgent cases of symptomatic or ruptured aneurysms. The necessity for off-the-shelf mbSG led to the production of the Zenith t-Branch device (Cook Medical, Bloomington, IN, USA), which according to studies, could be a treatment solution for over twothirds of patients requiring a stent graft [2,3]. However, target artery cannulation and connection with the bridging stents can be challenging in unfavourable anatomy.

The aim of the study is to present two cases in which the technique of unusual renal artery (RA) connection

Address for correspondence: Tomasz Jakimowicz, Department of General, Vascular and Transplant Surgery, Medical University of Warsaw,

Banacha Ia, 02-097, Warsaw, Poland, e-mail: tomj@wum.edu.pl 
was used to provide information that will help in cases of marginally suitable anatomy to bridge the renal arteries and allow endovascular treatment of symptomatic thoracoabdominal aneurysm.

\section{Case study}

Zenith $\mathrm{t}$-Branch is a one-size prosthesis that has four downward-oriented branches dedicated to the visceral arteries: celiac trunk, superior mesenteric artery, and both RAs. After adequate thoracic device placement, mbSG is introduced through femoral access. It is mandatory to position branches over the target vessels with an adequate gap. In typical fashion, vessels are catheterized antegrade with dedicated cuffs through axillary access. However, in cases of a steep angle of an RA, introducing a covered stent could be challenging or even impossible. If we manage to introduce at least a hydrophilic guidewire, it can be grabbed in the RA using a standard PTA balloon inserted into the RA through femoral access.

The surgery started through brachial access. After insertion of a long $(380 \mathrm{~cm})$ hydrophilic guidewire passing through the left brachial artery and femoral artery, the long I2F sheath was introduced through the left axillary artery. Then, thoracic endograft and a Zenith t-Branch were introduced, positioned, and deployed. Subsequently, we performed cannulation of the visceral arteries. In cases of steep angles of RAs (Fig. I), we cannulated and bridged the right RA from the contralateral branch to have a more convenient angle of the guidewires during cannulation. However, twice in the left RA, the manoeuvre was insufficient. Although we could cannulate it with guidewires, even a hydrophilic catheter slipped off during introduction, regardless of the type of guidewire used. We then introduced another guidewire and catheter to the left RA through left femoral access. Next, we advanced a $5 \mathrm{~mm} \times 2 \mathrm{~cm}$ PTA balloon over the wire into the vessel next to the guidewire introduced from above and through the branch. The balloon was expanded in the artery, thus grabbing the guidewire introduced from above (Fig. 2).

This manoeuvre grabbed the guidewire and prevented it from slipping out during catheter introduction. When the artery was catheterized and the balloon was re-expanded again, it was possible to introduce a stiffer wire (Rosen, Cook Medical) and bridge the artery with a Viabahn covered stent (WL Gore, Flagstaff, AZ, USA). Control angiography showed full patency of the stents without any kinking and proper nephrogram (Fig. 3).

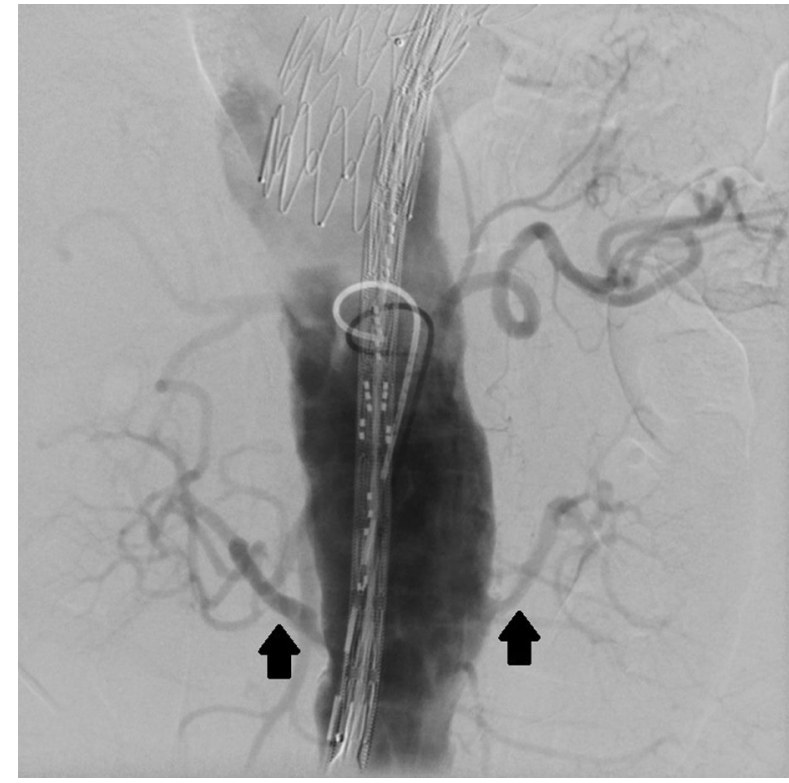

Figure I. Intraoperative fluoroscopy shows steep angles of renal arteries (arrows)

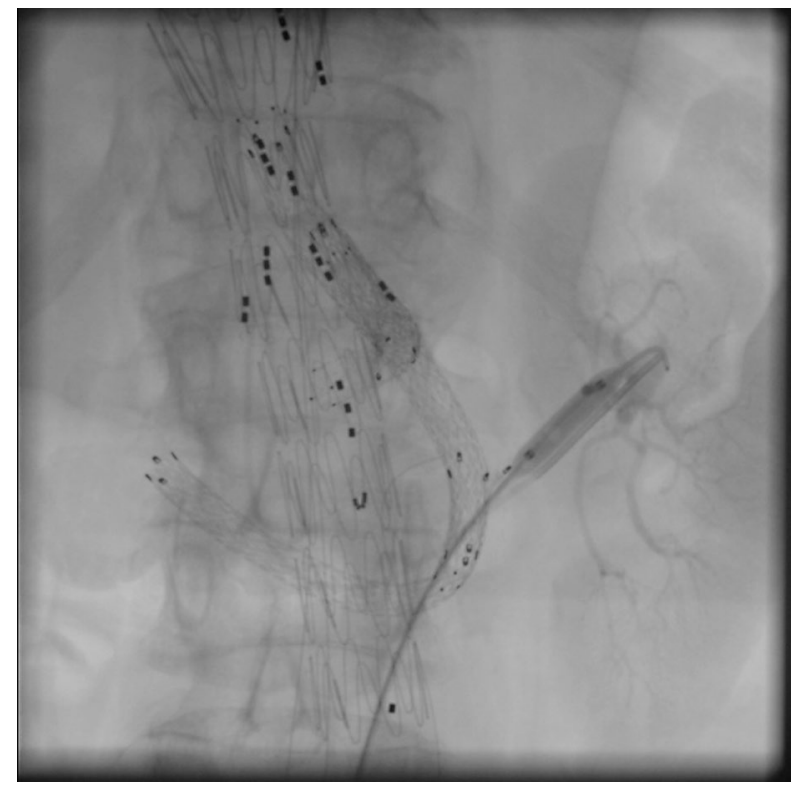

Figure 2. Expanded balloon grabs guidewire and prevents it from slipping out

Subsequently, the procedure was continued in the typical fashion. Total operation time, blood loss, radiation dose and radiation time are presented in Table $I$.

We used this technique in the cases of two patients who were admitted due to symptomatic TAAA. They were both unsuitable for open surgery due to comorbidities, and it was risky to wait for manufacture of 


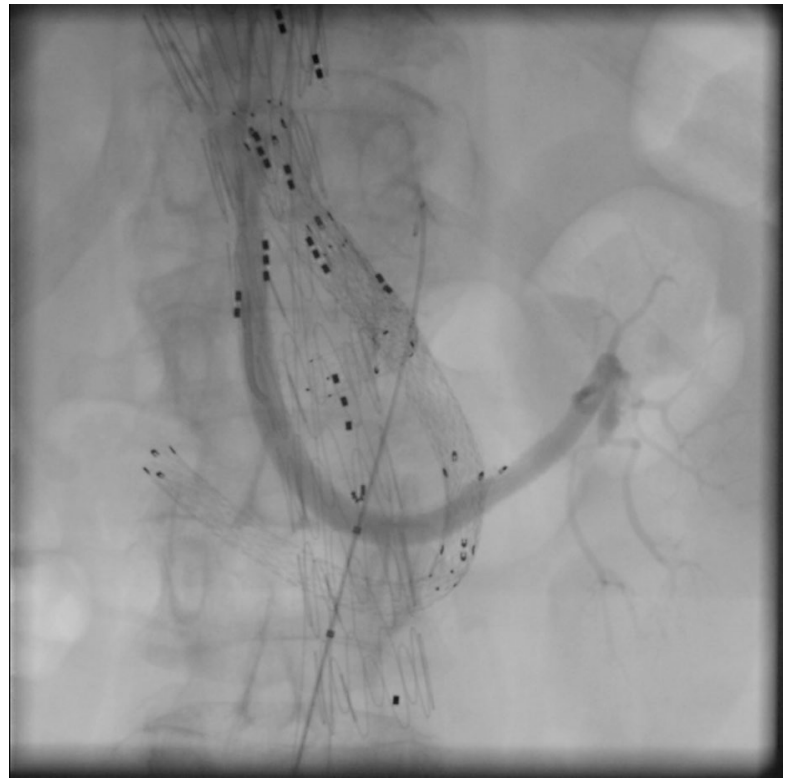

Figure 3. Control angiography with patent stent to the left kidney

Table I. Total operation time, radiation dose, radiation time and blood loss during procedures

\begin{tabular}{|l|c|c|}
\hline & Patient I & Patient 2 \\
\hline Total operation time $[\mathrm{min}]$ & 720 & 280 \\
\hline Radiation dose $\left[\mathrm{mGy} / \mathrm{m}^{2}\right]$ & 49.4 & 6.42 \\
\hline Radiation time $[\mathrm{min}]$ & 325.3 & 78.2 \\
\hline Blood loss $[\mathrm{mL}]$ & $\sim 2000$ & $\sim 500$ \\
\hline
\end{tabular}

a customized device. Therefore, we decided to use the Zenith t-Branch device, although steep-angle RAs were in opposition with its instruction for use [4].

For both patients, the postoperative period was not complicated, with routine 2-day intensive care unit stay for each. No deterioration of kidney function was observed. Unfortunately, Patient I had a basilar artery aneurysm that was unknown before surgery, and it ruptured on the third postoperative day. The rupture was complicated by pons ischemia and cerebral death. Patient 2's postoperative stay was uneventful, and the total postoperative hospital stay was 5 days. AngioCT at the 6-month follow-up showed patent branches without endoleak and no enlargement of the aneurysm diameter.

\section{Discussion}

TAAAs are relatively uncommon and represent only $3 \%$ of diagnosed aortic aneurysms according to stu- dies [5]. However, the complex anatomy of the disease demands proper planning of the treatment. Open repair was considered the "gold standard", despite the risk of significant perioperative morbidity and mortality observed even in the most experienced centres [6, 7]. The last decade saw the release of custom-made branched devices and, finally, the Zenith t-branch device, which subsequently received CE approval. From the very beginning, endovascular procedures with this endograft showed excellent early outcomes[8]. Moreover, Fernandez et al. compared EVAR using Zenith t-branch in the treatment of patients with suitable anatomy to EVAR using custom-made stent grafts. Midterm results were very similar, but waiting time for the former one was significantly shorter $(p<0.01)$ [9]. Thus, the endograft was considered to be a potential off-the-shelf prosthesis for TAAA. Feasibility studies suggest that it can be used to treat between $63 \%$ and $88 \%$ of cases of thoracoabdominal aneurysms with suitable anatomy $[3,10,11]$. Moreover, Chuter et al., from the aforementioned study, confirmed that during the study using off-the-shelf mbSG, their overall range of experience has increased and the scope of endovascular repair has expanded.

There are few published data on how to overcome intraoperative difficulties during an endovascular procedure with mbSG. Recently, Ferreira et al. presented a technique that is an endovascular bailout for catheterization in difficult visceral vessel anatomy [12]. However, the procedure requires an extra device not commonly used: the Indy Snare (Cook, Bloomington, IN, USA). This device has been effectively used in a small study group and was determined to be a valuable option. Oikonomou et al. proposed to cannulate the artery in retrograde through a small lumbotomy and grab the guidewire into the loop [13]. Although very effective, this manoeuvre increases invasiveness of the operation, which resulted in increased morbidity. Similarly to a previous case [14], we observed that the adjuvant manoeuvre permits a complete endovascular procedure, even outside of the instructions for use (IFU). To prevent kinking and acute ischemia of the kidneys, we decided to use flexible bridging stents from the contralateral side. What has to be admitted, Patient 2 had the procedure I month after Patient I, we had gained experience with this technique. Thus, Patient 2's surgery duration was three times shorter than that of Patient I. Blood loss, radiation time, and dose were also substantially less.

Spear and colleagues described how to perform EVAR in TAAA with multiple RAs [15]. To expand cri- 
teria for EVAR with steep angles of RA, it is possible to manufacture a custom-made device with upward branch. Still, the manufacturing of the device requires a couple of weeks, which is unacceptable in urgent cases. Our patients had symptomatic aneurysms, with rapid sac enlargement. Visceral anatomy of their aorta did not meet the criteria from IFU. Moreover, instruction for use states the precaution that $t$-branch was not evaluated on patients with symptomatic and ruptured TAAA.

\section{Conclusions}

Expansion of an additionally introduced balloon allows the guidewire in the RA to be grabbed. Therefore, aneurysm exclusion is possible. This technical note illustrates how endovascular RA revascularisation through mbSG can be safely performed in patients with unfavourable RA anatomy. The endovascular approach should be considered even in the presence of challenging anatomy in patients deemed unfit for surgery. Longer clinical follow-up and larger studies are required to better define the role of this technique.

\section{References}

I. Verhoeven ELG, Katsargyris A, Bekkema F, et al. Editor's Choice - Ten-year Experience with Endovascular Repair of Thoracoabdominal Aortic Aneurysms: Results from 166 Consecutive Patients. Eur J Vasc Endovasc Surg. 2015; 49(5): 524-53I, doi: 10.1016/j.ejvs.2014.11.018, indexed in Pubmed: 25599593.

2. Gasper WJ, Reilly LM, Rapp JH, et al. Assessing the anatomic applicability of the multibranched endovascular repair of thoracoabdominal aortic aneurysm technique. J Vasc Surg. 2013; 57(6): 1553-8; discussion 1558, doi: 10.1016/j.jvs.2012.12.021, indexed in Pubmed: 23395201.

3. Bisdas T, Donas KP, Bosiers M, et al. Anatomical suitability of the T-branch stent-graft in patients with thoracoabdominal aortic aneurysms treated using custom-made multibranched endografts. J Endovasc Ther. 2013; 20(5): 672-677, doi: 10.1583/134400MR. I, indexed in Pubmed: 24093320.

4. https://ifu.cookmedical.com/ifuPub/ReadFile?fileName=I FU-TBR_V7.PDF.

5. Stein L, Elefteriades J. Epidemiology and Natural History of Thoraco-Abdominal Aortic Aneurysms. Thoraco-Abdominal Aorta. 20I I: 25-32, doi: 10.1007/978-88-470-1857-0_3.
6. Nordon IM, Hinchliffe RJ, Holt PJ, et al. Modern treatment of juxtarenal abdominal aortic aneurysms with fenestrated endografting and open repair--a systematic review. Eur J Vasc Endovasc Surg. 2009; 38(I): 35-4I, doi: 10.10I6/j.ejvs.2009.02.012, indexed in Pubmed: 19346 |40.

7. Verhoeven ELG, Vourliotakis G, Bos WT, et al. Present and future of branched stent grafts in thoraco-abdominal aortic aneurysm repair: a single-centre experience. Eur J Vasc Endovasc Surg. 2009; 38(2): 155-161, doi: 10.1016/j.ejvs.2009.05.002, indexed in Pubmed: 19523863.

8. Bosiers MJ, Bisdas T, Donas KP, et al. Early experience with the first commercially available off-the-shelf multibranched endograft (t-branch) in the treatment of thoracoabdominal aortic aneurysms. J Endovasc Ther. 2013; 20(6): 719-725, doi: 10.1583/I3-4428R. I, indexed in Pubmed: 24325684.

9. Fernandez CC, Sobel JD, Gasper WJ, et al. Standard off-theshelf versus custom-made multibranched thoracoabdominal aortic stent grafts. J Vasc Surg. 2016; 63(5): 1208-1215, doi: 10.1016/j.jvs.2015.11.035, indexed in Pubmed: 268176I2.

10. Chuter TAM, Hiramoto JS, Park KH, et al. The transition from custom-made to standardized multibranched thoracoabdominal aortic stent grafts. J Vasc Surg. 20II; 54(3): 660-7; discussion 667, doi: 10.1016/j.jvs.2011.03.005, indexed in Pubmed: 21788114.

II. Sweet MP, Hiramoto JS, Park KH, et al. A standardized multibranched thoracoabdominal stent-graft for endovascular aneurysm repair. J Endovasc Ther. 2009; 16(3): 359-364, doi: 10.1583/09-2734. I, indexed in Pubmed: 19642795.

12. Ferreira M, Katsargyris A, Rodrigues E, et al. "Snare-Ride": A Bailout Technique to Catheterize Target Vessels With Unfriendly Anatomy in Branched Endovascular Aortic Repair. J Endovasc Ther. 2017; 24(4): 556-558, doi: 10.1 I77/I5266028।7709465, indexed in Pubmed: 285 I 4896

13. Oikonomou K, Katsargyris A, Brinster C], et al. Retrograde Target Vessel Catheterization as a Salvage Procedure in Fenestrated/Branched Endografting. J Endovasc Ther. 2015; 22(4): 603-609, doi: $10.1177 / 1526602815592205$, indexed in Pubmed: 26092541 .

14. Hammer P, Jakimowicz T, Romanowski L, et al. Application of Zenith t-Branch system in symptomatic thoracoabdominal aortic aneurysm with unfavourable anatomy - case report. Acta Angiologica. 2015; 21(2): 47-52, doi: 10.5603/aa.2015. 0009.

15. Spear R, Maurel B, Sobocinski J, et al. Technical note and results in the management of anatomical variants of renal vascularisation during endovascular aneurysm repair. Eur J Vasc Endovasc Surg. 2012; 43(4): 398-403, doi: 10.1016/j.ejvs.2012.01.003, indexed in Pubmed: 22306 I 75. 